\title{
HYPO-ANALYTIC PSEUDODIFFERENTIAL OPERATORS
}

\author{
S. BERHANU
}

(Communicated by Walter D. Littman)

\begin{abstract}
Let $\Omega$ be a hypo-analytic manifold of dimension $m$ equipped with a hypo-analytic structure whose structure bundle $T^{\prime}$ has dimension $m$. This paper introduces hypo-analytic pseudodifferential operators and it is shown that such operators preserve the hypo-analyticity of a distribution.
\end{abstract}

\section{INTRODUCTION}

The main concepts relating to hypo-analyticity were introduced by Baouendi, Chang, and Treves in [1], Chapter 1. We shall summarize some of these in this section.

Suppose $\Omega$ is a $C^{\infty}$ manifold of dimension $m+n$. A hypo-analytic structure on $\Omega$ is the data of an open covering $\left(U_{\alpha}\right)$ of $\Omega$ and for index $\alpha$, of $m C^{\infty}$ functions $Z_{\alpha}^{1}, \ldots, Z_{\alpha}^{m}$ satisfying the following two conditions:

(i) $d Z_{\alpha}^{\prime}, \ldots, d Z_{\alpha}^{m}$ are linearly independent at each point of $U_{\alpha}$;

(ii) if $U_{\alpha} \cap U_{\beta} \neq \varnothing$, there are open neighborhoods $\mathscr{O}_{\alpha}$ of $Z_{\alpha}\left(U_{\alpha} \cup U_{\beta}\right)$ and $\mathscr{O}_{\beta}$ of $Z_{\beta}\left(U_{\alpha} \cap U_{\beta}\right)$ and a holomorphic map $F_{\beta}^{\alpha}$ of $\mathscr{O}_{\alpha}$ onto $\mathscr{O}_{\beta}$, such that

$$
Z_{\beta}=F_{\beta}^{\alpha} \circ Z_{\alpha} \quad \text { on } \quad U_{\alpha} \cap U_{\beta} .
$$

When the $Z^{j}$ are real-valued and $n=0$, such a structure specializes to a real analytic structure. A distribution $h$ defined in an open neighborhood of a point $p_{0}$ of $\Omega$ is hypo-analytic at $p_{0}$ if there is a hypo-analytic local chart $\left(U_{\alpha}, Z_{\alpha \alpha}\right)$ whose domain contains $p_{0}$ and a holomorphic function $\tilde{h}_{c}$ defined on an open neighborhood of $Z_{c x}\left(p_{0}\right)$ in $C^{m}$ such that $h=\tilde{h}_{c} \circ Z$ in a neighborhood of $p_{0}$.

By a hypo-analytic local chart we mean an $(m+1)$-tuple $\left(U, Z^{1}, \ldots, Z^{m}\right)$ [abbreviated $(U, Z)$ ] consisting of an open subset $U$ of $\Omega$ and of $m$ hypoanalytic functions $Z^{1}, \ldots, Z^{m}$ whose differentials are linearly independent at every point of $U$.

Received by the editors November 2, 1987 and, in revised form, August 17, 1988.

1980 Mathematics Subject Classification (1985 Revision). Primary 35A20; Secondary 35S99. 
In general, the mapping $Z=\left(Z^{1}, \ldots, Z^{m}\right): U \rightarrow C^{m}$ is not a local embedding. However, when $\operatorname{dim} \Omega=m$, this mapping is a local diffeomorphism. Throughout this paper we will assume that the dimension of $\Omega$ is $m$.

\section{Preliminaries}

We will reason in a hypo-analytic local chart $(U, Z)$ in $\Omega$. We shall assume that the open set $U$ has been contracted sufficiently so that the mapping $Z=$ $\left(Z^{1}, \ldots, Z^{m}\right): U \rightarrow C^{m}$ is a diffeomorphism of $U$ onto $Z(U)$ and that $U$ is the domain of local coordinates $x_{j}(1 \leq j \leq m)$ all vanishing at a "central point" which will be denoted by 0 . We will suppose $Z(0)=0$ and denote by $Z_{x}$ the Jacobian matrix of the $Z^{j}$ with respect to the $x^{k}$. Substitution of $Z_{x}(0)^{-1} Z(x)$ for $Z(x)$ will allow us to assume that $Z_{x}(0)=$ the identity matrix. This will permit us to take the real part of the $Z^{j}(j=1, \ldots, m)$ as coordinates and write in these new coordinates

$$
Z^{j}=x^{j}+\sqrt{1} \Phi^{j}(x), \quad j=1, \ldots, m,
$$

where $\Phi=\left(\Phi^{1}, \ldots, \Phi^{m}\right)$ is real-valued whose differential at the origin is 0 . Moreover, the functions $Z^{j}$ are selected so that all the derivatives of order two of the $\Phi^{j}$ vanish at the origin. Indeed if this is not already so it suffices to replace each $Z^{j}$ by

$$
Z^{j}-\sqrt{-1} / 2 \sum \sum \frac{\partial^{2} \Phi^{j}}{\partial x^{k} \partial x^{l}}(0) Z^{k} Z^{l}
$$

We will use $Z_{x}^{*}$ to denote the transpose of the inverse of the matrix $Z_{x}$.

Since the first and second derivatives of all the $\Phi^{j}$ are zero at the origin, after contracting $U$ if necessary, we can find a number $c, 0<c<1$ such that for all $x, y \in U$ and for all $\xi \in R_{m}$,

$$
\left|\operatorname{Im} Z_{x}^{*}(x) \xi\right| \leq c\left|\operatorname{Re} Z_{x}^{*}(x) \xi\right|
$$

and

$$
\begin{aligned}
\operatorname{Re} & \left\{\sqrt{-1} Z_{x}^{*}(x) \xi \cdot(Z(x)-Z(y))-<Z_{x}^{*}(x) \xi>(Z(x)-Z(y))^{2}\right\} \\
& \leq-c|\xi||Z(x)-Z(y)|^{2}, \quad \text { where }\langle\zeta\rangle=\left(\zeta_{1}^{2}+\cdots+\zeta_{m}^{2}\right)^{1 / 2}
\end{aligned}
$$

\section{HYPO-ANALYTIC PSEUDODIFFERENTIAL OPERATORS}

Our aim is to introduce pseudodifferential operators that are naturally associated with hypo-analytic structures. This definition generalizes analytic pseudodifferential operators (for a treatment of the analytic theory see [6]).

Definition 3.1. Let $d$ be a real number. We denote by $\tilde{S}^{d}(U, U)$ the space of holomorphic functions $\tilde{a}(z, w, \theta)$ in a product set $\mathscr{O} \times \mathscr{O} \times \mathscr{C}$ with $\mathscr{O}$ an open neighborhood of $Z(U)$ and $\mathscr{C}$ an open cone in $C_{m} \backslash\{0\}$ containing $R_{m} \backslash\{0\}$, which have the following property: 
Given any compact subset $K$ of $\mathscr{O}$ and any closed cone $\mathscr{C}^{\prime} \subset \mathscr{C}$ whose interior contains $R_{m} \backslash\{0\}$, there is a constant $r>0$ such that for all $z, w$ in $K$ and all $\theta$ in $\mathscr{C}^{\prime}$, we have :

$$
|\tilde{a}(z, w, \theta)| \leq r(1+|\theta|)^{d} .
$$

Definition 3.2. We say that a $C^{\infty}$ function $a(x, y, \theta)$ in $U \times U \times R_{m}$ is a hypo-analytic amplitude of degree $d$ and we write $a \in S^{d}(U, U)$ if there is $\tilde{a} \in \tilde{S}^{d}(U, U)$ such that

$$
a(x, y, \theta)=\tilde{a}(Z(x), Z(y), \theta), \quad \text { for all } x \text { in } U, \quad y \text { in } U, \quad 0 \neq \theta \in R_{m} \text {. }
$$

Let $a(x, y, \theta)=\tilde{a}(Z(x), Z(y), \theta)$ be a hypo-analytic amplitude of degree $d \in R$ in $U \times U$. For any $\varepsilon>0$ and $u \in C_{c}^{0}(U)$ we define the linear operator $A^{\varepsilon} u(x)$

$$
=\left(\frac{1}{4 \pi^{3}}\right)^{m / 2} \int_{U} \int_{R_{m}} \exp \left(\sqrt{-1} \xi \cdot\left(Z(x)-Z(y)-\varepsilon|\xi|^{2}\right) a(x, y, \xi) u(y) d Z(y) d \xi .\right.
$$

We contract $U$ sufficiently so that for every $x, y \in U$ and $\xi \in R_{m}$ the point $Z_{x}^{*}(x) \xi+\sqrt{-1}\left\langle Z_{x}^{*}(x) \xi\right\rangle(Z(x)-Z(y))$ will remain in the cone in which $a(x, y, \cdot)$ is defined. We observe that each $A^{\varepsilon} u$ is a hypo-analytic function.

Theorem 3.1. When $\varepsilon \rightarrow 0, A^{\varepsilon}$ converges to a continuous linear operator A: $E^{\prime}(U) \rightarrow \mathscr{D}^{\prime}(U)$ which maps $C_{c}^{\infty}(U)$ into $C^{\infty}(U)$ continuously.

Proof. We deform the path of $\xi$-integration from $R_{m}$ to the image of $R_{m}$ under the map

$$
\xi \rightarrow \zeta(\xi)=Z_{x}^{*}(x) \xi+\sqrt{-1}\left\langle Z_{x}^{*}(x) \xi\right\rangle(Z(x)-Z(y))
$$

Thus

$A^{\varepsilon} u(x)$

$$
\begin{aligned}
&=\left(\frac{1}{4 \pi^{3}}\right)^{m / 2} \int_{U} \int_{R_{m}} \exp \left(\sqrt{-1} Z_{x}^{*}(x) \xi \cdot(Z(x)-Z(y))\right. \\
&-\left\langle Z_{x}^{*}(x) \xi\right\rangle(Z(x)-\left.Z(y))^{2}-\varepsilon\langle\zeta(\xi)\rangle^{2}\right) \\
& \times a(x, y, \zeta(\xi)) u(y) \operatorname{det}\left(\frac{\partial \zeta}{\partial \xi}\right) d Z(y) d \xi .
\end{aligned}
$$

If the amplitude $a$ has degree $d<-m-1$ and $u \in C_{c}(U)$, condition (2.1) will imply that $A^{\varepsilon} u$ converges uniformly on compact subsets of $U$ to a continuous function $A u$. Moreover, in this case, $A: C_{c}^{0}(U) \rightarrow C^{0}(U)$ will be a continuous operator.

In general, if the degree of $a=d$, we consider the holomorphic functions $\widetilde{A}^{\varepsilon} u(z)$

$$
=\left(\frac{1}{4 \pi^{3}}\right)^{m / 2} \int_{U} \int_{R_{m}} \exp \left(\sqrt{-1} \xi \cdot(z-Z(y))-\varepsilon|\xi|^{2}\right) \tilde{a}(z, Z(y), \xi) u(y) d Z(y) d \xi .
$$


We denote the Laplacian $\sum_{j=1}^{m} D_{z_{j}}^{2}$ by $\Delta_{z}$ and write $\widetilde{A}^{\varepsilon} u(z)$

$=\left(\frac{1}{4 \pi^{3}}\right)^{m / 2} \int_{U} \int_{R_{m}}\left(1-\Delta_{z}\right)^{k}\left\{e^{\sqrt{-1} \xi(z-Z(y))-\varepsilon|\xi|^{2}}\right\} \frac{\tilde{a}}{\left(1+|\xi|^{2}\right)^{k}} u(y) d Z(y) d \xi$.

In the latter, we apply the transposed Leibniz formula to get

$$
\begin{aligned}
\left\{\left(1-\Delta_{z}\right)^{k} e^{\sqrt{-1} \xi(z-\omega)}\right\} \tilde{a}(z, \omega, \xi) & \\
= & \sum_{|\alpha+\beta| \leq 2 k} c_{\alpha, \beta}\left(\frac{\partial}{\partial z}\right)^{\alpha}\left[e^{\sqrt{-1} \xi(z-\omega)}\left(\frac{\partial}{\partial z}\right)^{\beta} \tilde{a}(z, \omega, \xi)\right],
\end{aligned}
$$

where the $c_{\alpha, \beta}$ are integers.

We can thus write $A^{\varepsilon} u(x)=\sum_{|\alpha| \leq 2 k} M^{\alpha}\left(A_{\alpha}^{\varepsilon} u(x)\right)$, where the $A_{\alpha}^{\varepsilon}$ are defined like $A^{\varepsilon}$ except that their amplitudes have degree $\leq d-2 k$. For $k$ sufficiently large we have shown that $A^{\varepsilon} u$ converges to a continuous function. Therefore, $A^{\varepsilon} u$ converges to $A u$ in the space $\mathscr{D}^{\prime}(U)$. Suppose now $u \in \mathscr{E}^{\prime}(U)$. We choose continuous functions $u_{\alpha} \in C_{c}^{0}(U)$ that satisfy $u=\sum_{|\alpha| \leq k} M^{\alpha} u_{\alpha}$. We may integrate by parts to get, for each $\alpha, A^{\varepsilon}\left(M^{\alpha} u_{\alpha}\right)=A_{\alpha}^{\varepsilon}\left(u_{\alpha}\right)$, where the degree of the amplitude of $A_{\alpha}^{\varepsilon}$ is $\leq|\alpha|+d$. Thus $A^{\varepsilon} u=\sum_{|\alpha| \leq k} A_{\alpha}^{\varepsilon}\left(u_{\alpha}\right)$ which brings us to a situation already considered. We conclude that $A^{\varepsilon} u \rightarrow A u$ in $\mathscr{D}^{\prime}(U)$.

Suppose now $u \in C_{c}^{\infty}(U)$. We denote $\sum_{j=1}^{m} M_{j}^{2}$ by $\Delta_{M}$, where the $M_{j}$ are vector fields satisfying $M_{j} Z^{k}=\delta_{j}^{k}$. Integration by parts gives

$$
\begin{aligned}
A^{\varepsilon} u(x)=\left(\frac{1}{4 \pi^{3}}\right)^{m / 2} \int_{U} \int_{R_{m}} \exp \left(\sqrt{-1} \xi \cdot(Z(x)-Z(y))-\varepsilon|\xi|^{2}\right) \\
\times \frac{\left(1-\Delta_{M}\right)^{k}\{a(x, y, \xi) u(y)\}}{\left(1+|\xi|^{2}\right)^{k}} d Z(y) d \xi .
\end{aligned}
$$

After deforming contour as in (3.1), we see that $A^{\varepsilon} u$ converges in $C_{c}^{0}(U)$ to the continuous function $A u$. Moreover, the same convergence also occurs for $M^{\alpha}\left(A^{\varepsilon} u\right)$ for all $\alpha$. It follows that $A u \in C^{\infty}(U)$. Finally, we will show that the operator $A: C_{c}^{\infty}(U) \rightarrow C_{c}^{\infty}(U)$ is continuous. Let $u \in C_{c}^{\infty}(U)$. We can write (3.2) as

(3.3) $A u(x)=$

$$
\begin{array}{r}
\left(\frac{1}{4 \pi^{3}}\right)^{m / 2} \int_{U} \int_{R_{m}} \exp \left(\sqrt{-1} Z_{x}(x) \xi \cdot(Z(x)-Z(y))-\left\langle Z^{*}(x) \xi\right\rangle(Z(x)-Z(y))^{2}\right) \\
\times \frac{\left(1-\Delta_{M}\right)^{k}\{a(x, y, \zeta(\xi)) u(y)\}}{\left(1+|\xi|^{2}\right)^{k}} \operatorname{det} \frac{\partial \zeta}{\partial \xi} d Z(y) d \xi .
\end{array}
$$

We note that both the exponential term and $\operatorname{det} \partial \zeta / \partial \xi$ are bounded. Suppose now the sequence $u_{n} \rightarrow u$ in $C_{c}^{\infty}(U)$. Then (3.3) shows that $A u_{n} \rightarrow A u$ in 
$C_{c}^{0}(U)$. To conclude the proof, it suffices to show that for every multi-index $\alpha$, the sequence $M^{\alpha}\left(A u_{n}\right)$ is uniformly convergent on compact subsets of $U$. For each $\alpha$, there is an amplitude $b^{\alpha}$ of degree $\leq d+|\alpha|$ such that

$$
\begin{aligned}
& M^{\alpha}\left(A^{\varepsilon} u\right)(x) \\
& =\left(\frac{1}{4 \pi^{3}}\right)^{m / 2} \int_{R_{m}} \int_{U} \exp \left(\sqrt{-1} \xi \cdot(Z(x)-Z(y))-\varepsilon|\xi|^{2}\right) b^{\alpha}(x, y, \xi) u(y) d Z(y) d \xi .
\end{aligned}
$$

By what we have already seen, the right-hand side converges in the space $C_{c}^{0}(U)$.

Definition 3.3. The operator $A: \mathscr{E}^{\prime}(U) \rightarrow \mathscr{D}^{\prime}(U)$ of Theorem 3.1 will be called a hypo-analytic pseudodifferential operator.

Example. A hypo-analytic differential operator on $\Omega$ may be defined as a linear differential operator $P$ on $\Omega$ satisfying the following property:

For every open subset $\Omega^{\prime}$ of $\Omega$ and every hypo-analytic function $f$ on $\Omega^{\prime}$, Pf is hypo-analytic on $\Omega^{\prime}$. In the hypo-analytic chart $(U, Z)$, let $M_{j}(1 \leq$ $j \leq m)$ be the vector fields satisfying

$$
M_{j} Z^{k}=\delta_{j}^{k}
$$

Then a hypo-analytic differential operator $P$ takes the form

$$
P=\sum_{|\alpha| \leq k} a_{\alpha} M^{\alpha},
$$

where each $a_{\alpha}$ is a hypo-analytic function on $U$. We will show that such an operator is an example of a hypo-analytic pseudodifferential operator. For $u \in E^{\prime}(U)$ and $\varepsilon>0$ let

$$
u^{\varepsilon}(x)=\left(\frac{1}{4 \pi^{3}}\right)^{m / 2} \int_{R_{m}} \int_{U} \exp \left(\sqrt{-1} \xi \cdot(Z(x)-Z(y))-\varepsilon|\xi|^{2}\right) u(y) d Z(y) d \xi .
$$

In [1], the authors observed that $u^{\varepsilon} \rightarrow u$ in the space $\mathscr{D}^{\prime}(U)$. Write $P$ as $\sum_{|\alpha| \leq k} b_{\alpha} N^{\alpha}$ where each $b_{\alpha}$ is hypo-analytic and $N_{j}=-\sqrt{-1} M_{j}$ for each $j$. We then have

$$
N_{j} u^{\varepsilon}(x)=\left(\frac{1}{4 \pi^{3}}\right)^{m / 2} \int_{R_{m}} \int_{U} \exp \left(\sqrt{-1} \xi \cdot(Z(x)-Z(y))-\varepsilon|\xi|^{2}\right) \xi_{j} u(y) d Z d \xi
$$

for each $j$ and therefore

$$
\begin{gathered}
P u^{\varepsilon}(x)=\left(\frac{1}{4 \pi^{3}}\right)^{m / 2} \int_{R_{m}} \int_{U} \exp \left(\sqrt{-1} \xi \cdot(Z(x)-Z(y))-\varepsilon|\xi|^{2}\right) \\
\times\left(\sum_{|\alpha| \leq k} b_{\alpha}(x) \xi^{\alpha}\right) u(y) d Z d \xi
\end{gathered}
$$


When $\varepsilon \rightarrow 0$, we get $P u=A u$, where $A$ is the hypo-analytic pseudodifferential operator whose amplitude is

$$
\sum_{|\alpha| \leq k} b_{\alpha}(x) \xi^{\alpha}
$$

\section{PSEUdolocal PROPERTY}

The aim of this section is to show that hypo-analytic pseudodifferential operators map hypo-analytic functions to hypo-analytic functions.

Since the first and second derivatives of $\Phi$ vanish at the origin, after shrinking $U$ if necessary, we may assume that for all $x, y$ in $U$,

$$
|\Phi(x)-\Phi(y)| \leq|x-y| / 2
$$

and

$$
|\Phi(y)| \leq 1 / 2|y|^{2}
$$

We shall need the following lemma.

Lemma 4.1. Let $A$ be a hypo-analytic pseudodifferential operator with amplitude $a(x, y, \xi)=\tilde{a}(Z(x), Z(y), \xi)$ and let $u$ be in $E^{\prime}(U)$. If $u$ vanishes in some neighborhood of $0, A u$ is hypo-analytic at 0 .

Proof. For each $\varepsilon>0$ we consider the holomorphic function

$\widetilde{A}^{\varepsilon} u(z)$

$$
=\left(\frac{1}{4 \pi^{3}}\right)^{m / 2} \int_{U} \int_{R_{m}} \exp \left(\sqrt{-1} \xi(z-Z(y))-\varepsilon|\xi|^{2}\right) \tilde{a}(z, Z(y), \xi) u(y) d Z(y) d \xi .
$$

We deform the path of $\xi$-integration from $R_{m}$ to the image of $R_{m}$ under the map $\zeta(\xi)=\xi+\sqrt{-1}|\xi|(z-Z(y))$ and write

$$
\begin{aligned}
\widetilde{A}^{\varepsilon} u(z)=\left(\frac{1}{4 \pi^{3}}\right)^{m / 2} & \int_{U} \int_{R_{m}} \exp \left(\sqrt{-1} \xi(z-Z(y))-|\xi|(z-Z(y))^{2}-\varepsilon\langle\zeta(\xi)\rangle\right) \\
& \times \tilde{a}(z, Z(y), \zeta(\xi)) u(y) \frac{\partial \zeta}{\partial \xi} d Z(y) d \xi
\end{aligned}
$$

Let $Q(z)=\operatorname{Re}\left\{\sqrt{-1} \xi \cdot(z-Z(y))-|\xi|(z-Z(y))^{2}\right\}$. Using (4.1) we have $Q(0)=\xi \cdot \phi(y)-|\xi|\left(|y|^{2}-|\phi(y)|^{2}\right) \leq-\frac{1}{4}|y|^{2}|\xi|$. Let $d$ be a positive number such that $y \in \operatorname{supp} u \Rightarrow|y|>d$. Then $Q(0) \leq-\frac{1}{4} d^{2}|\xi|$ which by continuity implies that $Q(z) \leq-\frac{1}{3} d^{2}|\xi|$ for $z$ in a sufficiently small neighborhood of 0 . Therefore, as $\varepsilon \rightarrow 0, \widetilde{A^{\varepsilon}} u(z)$ converges uniformly on some neighborhood of 0 . It follows that $A u(x)=\tilde{A} u(Z(x))$ is hypo-analytic at 0 . 
Theorem 4.2. Suppose $A$ is a hypo-analytic pseudodifferential operator and $u \in$ $\mathscr{E}^{\prime}(U)$. If $u$ is hypo-analytic at 0 then $A u$ is hypo-analytic at 0 .

Proof. Let $\tilde{u}$ be a holomorphic function such that $u(y)=\tilde{u}(Z(y))$ for $y$ near 0 . In the integral for $\widetilde{A}^{\varepsilon} u(z)$ we deform the " $y$-contour" from $U$ to the image of $U$ under the map $Z(y) \rightarrow \widetilde{Z}(y)=Z(y)-\sqrt{-1} \chi(y) d \xi /|\xi|$, where $d$ is a sufficiently small positive number, $\chi \in C_{c}^{\infty}(U), 0 \leq \chi \leq 1, \chi \equiv 1$ near 0 and $\operatorname{supp} \chi$ sufficiently small. We may thus write

$$
\begin{gathered}
\widetilde{A}^{\varepsilon} u(z)=\left(\frac{1}{4 \pi^{3}}\right)^{m / 2} \int_{R_{m}} \int_{U} \exp \left(\sqrt{-1} \xi(z-Z(y))-d \chi(y)|\xi|-\varepsilon|\xi|^{2}\right) \\
\times a(z, \widetilde{Z}(y), \xi) \tilde{u}(\tilde{Z}(y)) d \tilde{Z}(y) d \xi .
\end{gathered}
$$

We next deform the $\xi$-integration to the image of $R_{m}$ under the map $\xi \rightarrow$ $\zeta(\xi)=\xi+\sqrt{-1}|\xi|(z-Z(y))$. We will show that $\widetilde{A}^{\varepsilon} u(z)$ converges uniformly near $z=0$. To prove this, we will estimate

$$
Q(z)=\operatorname{Re}\left\{\sqrt{-1} \xi(z-Z(y))-|\xi|(z-Z(y))^{2}-d \chi(y)\langle\zeta(\xi)\rangle\right\} .
$$

Lemma 4.1 allows us to shrink the support of $u$ so that when $y \in \operatorname{supp} u$ and $z$ is small enough, $|\xi| / 2 \leq \operatorname{Re}\langle\zeta(\xi)\rangle$. Moreover, for such $z$ and $y$ we have: $\left.\operatorname{Re}\left\{\sqrt{-1} \xi(z-Z(y))-|\xi|(z-Z(y))^{2}\right\} \leq\left(-|y|^{2} / 8+3|z|\right)|\xi|\right)$. Therefore for $z$ near $0, Q(z) \leq-\left(|y|^{2} / 8+d \chi(y) / 2-3|z|\right)|\xi|$. This estimate together with the fact that $\chi(y) \equiv 1$ near $y=0$ yield the required result.

In [1] the authors microlocalized hypo-analyticity by first adapting Sato's definition and then showing its equivalence with the one derived from the FourierBros-Iagolnitzer transform [4]. The operators defined in this paper also preserve microlocal hypo-analyticity [3].

\section{ACKNOWLEDGMENTS}

I wish to express my gratitude to Professor Francois Treves for his advice and encouragement during his direction of my dissertation [2] which is an offspring of [1] and [5].

\section{REFERENCES}

1. M. S. Baouendi, C. H. Chang and F. Treves, Microlocal hypo-analyticity and extensions of $C R$ functions, J. Differential Geom. 18 (1983), 331-391.

2. S. Berhanu, Hypo-analytic pseudodifferential operators, Thesis, Rutgers University, 1987.

3. Microlocal hypo-analyticity and hypo-analytic pseudodifferential operators, Proc. Amer. Math. Soc., (to appear).

4. J. Bros and D. Iagolnitzer, Support essentiel et structure analytique des distributions, Séminarie Goulaouic-Lions-Schwartz, 1975-76. No. 18.

5. F. Treves, Hypo-analytic structures (in preparation).

6. __ Introduction to pseudodifferential and Fourier integral operators, Volume I, Plenum Press, New York, 1980.

Current address: Department of Mathematics, Temple University, Philadelphia, Pa. 